\title{
Structure of Nascent Microbial Cellulose IV. Influence of Size and Substitution Groups of Direct Dye on Nascent Microbial Cellulose
}

\author{
Hiroyasu $\mathrm{KIDO}^{\dagger}$ and Akira $\mathrm{KAI}$ \\ Department of Industrial Chemistry, Tokyo Metropolitan University, \\ Minami-Ohsawa 1-1, Hachioji-shi, Tokyo 192 0397, Japan
}

(Received December 3, 1997)

\begin{abstract}
The structures of products obtained from Acetobacter-cultures in the presence of direct dyes and cellulose regenerated from those were examined by X-ray measurements. The products obtained in the presence of direct dyes showed the X-ray diffraction diagrams with two distinct peaks different from the diffraction diagram of microbial cellulose. These products were crystalline complexes containing the direct dye between the cellulose sheets corresponding to the (1Ti0) plane of microbial cellulose. Cellulose I was regenerated from the Direct Brown 106 and the Direct Blue 71 complexes, cellulose IV was regenerated from the Direct Red 28 complex and cellulose II was regenerated from the Direct Red 80 complex. The different effects of direct dyes on the structure of the nascent cellulose may be due mainly to the number of sulfonate groups per unit of molecular weight.

KEY WORDS Acetobacter xylinum / Microbial Cellulose / Direct Dye / Dye-Cellulose Complex / Cellulose I / Cellulose II / Cellulose IV /
\end{abstract}

Acetobacter xylinum (A. xylinum) produces extracellular cellulose I $\alpha$ rich cellulose. ${ }^{1}$ However, this nascent cellulose is in a noncrystalline state. ${ }^{2}$ Accordingly, various substances, such as medium components, ${ }^{3}$ heavy metal salts, ${ }^{4}$ and the direct dye for cellulose ${ }^{5-8}$ diffuse into the nascent cellulose. Therefore, microbial cellulose (MC) with different fine structures can be prepared from interactions of the diffusion substance and nascent cellulose according to characteristics of the diffusion substance.

The crystalline complex composed of a dye and cellulose, obtained from $A$. xylinum-culture in the presence of Fluorescent Brightener, is transformed into cellulose I by dye-extraction. ${ }^{5-10}$ This cellulose is not a cellulose $\mathrm{I} \alpha$ rich structure for ordinary MC but cellulose $\mathrm{I} \beta$ rich structure. ${ }^{11}$ Moreover, cellulose IV is regenerated from the complex produced in the presence of Direct Red 28. ${ }^{12,13}$ Cellulose II is regenerated from complexes produced in the presence of Direct Blue 1, 14, 15, or $53^{13-15}$ having the same biphenylenebis(azo) skeletal structure as Direct Red 28, Direct Red 75 or $79^{16}$ having ureylenebis(naphthylazo)benzene skeletal structure. But, there are slight differences in the structures of these regenerated celluloses II. Although the structures of regenerated celluloses depend on the structures and properties of dyes, the main factor is not always clear.

This paper reports the influence of dyes on the structure of nascent MC, judging from structures of the products and regenerated celluloses obtained by $A$. xylinum-cultures in the presence of various dyes. Such dyes are Direct Brown 106 (DBr106) with the longest molecular length in a direct dye, Direct Red 28 (DR28) with the shortest molecular length, Direct Red 80 (DR80) with a molecular weight somewhat larger than DBr106 and having two more sulfonate groups than $\mathrm{DBr} 106$ or Direct Blue 71 (DB71).

\section{EXPERIMENTAL}

\section{Preparation of Samples}

Product Sample. Cultivation was performed by the methods in previous papers. ${ }^{14-16}$ A. xylinum (IFO 13693) was cultured in a $15 \mathrm{~cm}$ diameter petridish containing $110 \mathrm{ml}$ Hestrin-Schramm medium ${ }^{17}$ (pH 6.8) at $28.0^{\circ} \mathrm{C}$ for 3 days in an incubator. $60 \mathrm{ml}$ of cellulosefree cell suspension, prepared from matured petridish in $120 \mathrm{ml}$ phosphate buffer solution, were added to $140 \mathrm{ml}$ of HS medium ( $\mathrm{pH} 7.0$ ) containing a direct dye of $0.1 \mathrm{wt} \%$ and the mixture was incubated at $28.0^{\circ} \mathrm{C}$ for $24 \mathrm{~h}$ under static conditions. The product was collected by centrifugation (6000 rpm, $5.15 \mathrm{~g}, 20 \mathrm{~min}$ ) and washed well with $0.1 \mathrm{wt} \%$ aqueous $\mathrm{NaOH}$ solution to remove the dye not related to dyeing and then washed thoroughly with distilled water until alkali-free. These products were preserved at about $3^{\circ} \mathrm{C}$ in a wet state.

The direct dyes used in this work are DB71

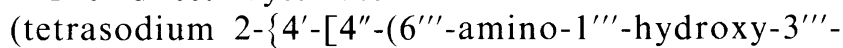
sulfonato-2"' -naphthylazo)-6" -sulfonato- $1^{\prime \prime}$-naphthylazo]-1'-naphthylazo -naphthalene-4,8-disulfonate, Sumitomo Chemicals), DBr106 (tetrasodium $N, N^{\prime}$-bis\{3-methyl-4- $\left\{3^{\prime}\right.$-methyl-4'-[2"'sulfonato-4" - $\left(4^{\prime \prime \prime}\right.$-sulfonatophenylazo)phenylazo]phenylazo phenyl $\}$ urea, Sumitomo Chemicals), DR28 (2,2'-[4,4'-biphenylenebis(azo)] bis[1-amino-naphthalene-4-sulfonic acid]disodium salt, Aldrich Chemical), DR80 (hexasodium $N, N^{\prime}$-bis $\{1$-hydroxy-2-[2'-sulfonato-4'-(4"'sulfonatophenylazo)phenylazo]-3-sulfonato-6-naphthyl \}urea, Ciba-Gaigy). The chemical structures of these dyes are shown in Figure 1.

DB71 and DR28 were purified by salting out. $1 \mathrm{wt} \%$ aqueous solution of a dye was heated at $80^{\circ} \mathrm{C}$ and filtered to reject insoluble matter. $40 \mathrm{wt} \%$ aqueous sodium acetate solution in a ratio of 1 to 5 was added to the filtrate with agitation at $80^{\circ} \mathrm{C}$ and settled until it reaches to the room temperature. The sedimental dye was

\footnotetext{
${ }^{\dagger}$ Present address: Mitsui Toatsu Chemicals Inc., Kasumigaseki 3-2 5, Chiyoda-ku, Tokyo 100 0013, Japan.
} 


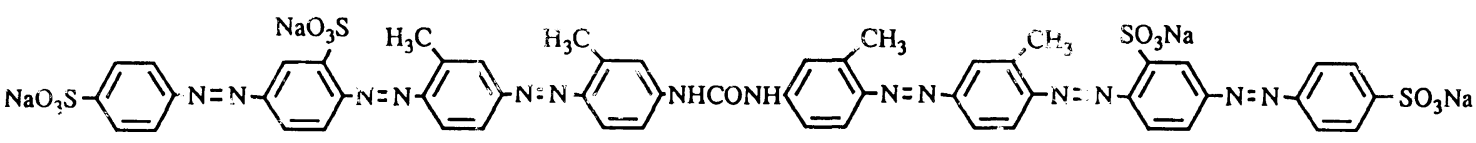

Direct Brown 106

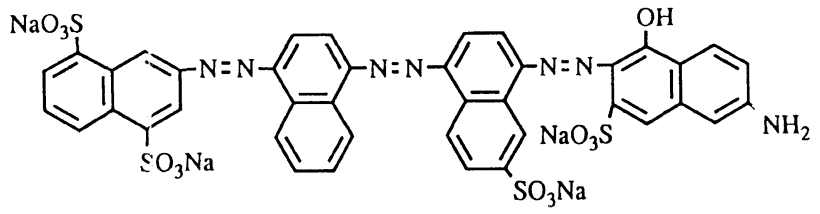

Direct Blue 71<smiles>Nc1c(N=Nc2ccc(-c3ccc(N=Nc4cc([N+](=O)[O-])c5ccccc5c4N)cc3)cc2)cc([N+](=O)[O-])c2ccccc12</smiles>

Direct Red 28

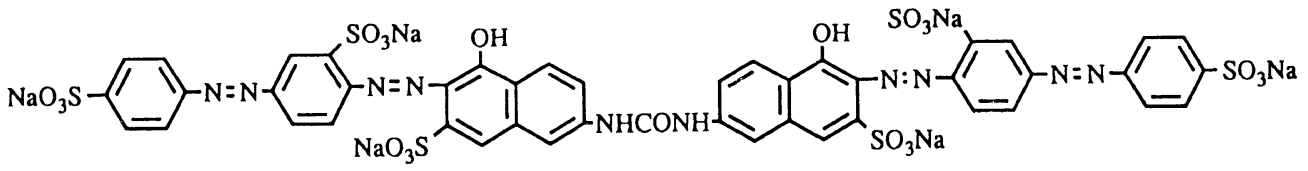

\section{Direct Red 80}

Figure 1. Chemical structures of direct dyes.

collected on the glass filter and washed with hot ethanol until it disappears. The dye was refined by repeating the operation three times and dried over $\mathrm{P}_{2} \mathrm{O}_{5}$ under reduced pressure. DBr106 and DR80 were purified by recrystallization. The dye was added to the mixed solvent of ethanol and water (in case of DBr106, EtOH : $\mathrm{H}_{2} \mathrm{O}=$ $90: 10$, in case of DR80, EtOH $: \mathrm{H}_{2} \mathrm{O}=70: 30$ ) and dissolved at $70^{\circ} \mathrm{C}$ and filtered. The filtrate was left in static condition to recrystallize the dye. The dye was refined by repeating the operation three times and dried over $\mathrm{P}_{2} \mathrm{O}_{5}$ under reduced pressure.

Regenerated Cellulose Sample. The dye-extraction of the above products was performed by boiling in $70 \mathrm{vol} \%$ ethanol solution for $18 \mathrm{~h}$ and fresh aqueous ethanol solution was exchanged every $3 \mathrm{~h}$. The dye-extracted sample was again boiled in $1.0 \mathrm{wt} \%$ aqueous sodium hydroxide solution for $10 \mathrm{~h}$ under $\mathrm{N}_{2}$ atomosphere. The regenerated cellulose sample was neutralized with 1.0 vol\% aqueous acetic acid and rinsed well with distilled water. The sample was preserved as above.

\section{Methods for Measurement}

$X$-Ray Measurement. This was done as in previous papers. ${ }^{14-16}$ The X-ray diffraction diagram of an uniplanar oriented membrane of the product was measured by reflection and transmission. The membranes were prepared from the preserved product, which was dried on a Teflon plate at atmospheric conditions. The diffraction diagrams of an isotropic product and re- generated cellulose were made by transmission. In this method, the sample was compressed as a pellet into the pore of a sample holder of $2 \mathrm{~mm}$ in diameter and $1 \mathrm{~mm}$ in thickness. The X-ray diffractogram was measured by a $\mathrm{MXP}^{18}$ diffractometer (MAC Science) with Ni-filtered $\mathrm{Cu}-K_{\alpha}$ radiation. Measurement conditions were as follows: divergence slit, $1.0 \mathrm{~mm}$; receiving slit, $0.15 \mathrm{~mm}$; scanning speed, $4.0^{\circ} \mathrm{min}^{-1}$; X-ray radiation, $40 \mathrm{kV}$, $200 \mathrm{~mA}$.

\section{RESULTS AND DISCUSSION}

Figure 2 shows X-ray diffractograms of isotropic MC and the isotropic product obtained from $A$. xylinumculture in the presence of DB71, DBr106, DR28, or DR80 (DB71 product, DBr106 product, DR28 product, and DR80 product). $2 \theta \mathrm{s}$ of diffraction peaks of each diagram are listed in Table I.

In all cases, the products obtained in the presence of a direct dye showed characteristic diagrams with two diffraction peaks: one occurs strongly at $20-23^{\circ}$ and the others weakly at $9^{\circ}$ to $17^{\circ}$. The diffractogram of the DR80 product was somewhat different from the other three diagrams because the peak at high angle side occurs near $20^{\circ}$. The diffractogram of the DR80 product is similar to the diagrams of the DB1, 14, 15, 53, DR75, and 79 products as reported previously. ${ }^{14-16}$ These four diffractograms of the product having two peaks, one at high angle side and the other at low angle side, are similar 


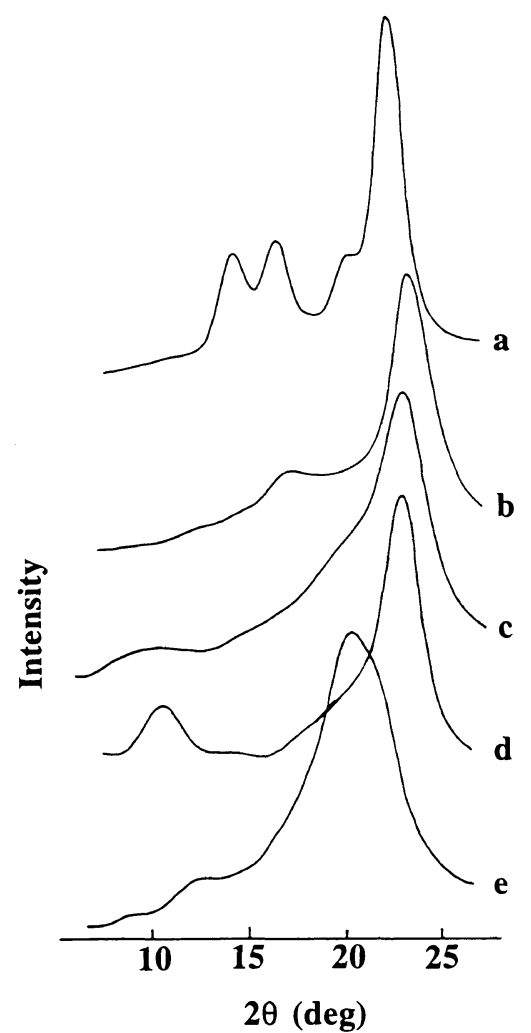

Figure 2. X-Ray diffractograms of microbial cellulose and product samples from Acetobacter-culture in the presence of a dye of $0.1 \mathrm{wt} \%$. a, MC; b, DB71 product; c, DBr106 product; d, DR28 product; e, DR80 product.

Table I. X-Ray diffraction angles of $\mathrm{MC}$ and products obtained from Acetobacter-culture in the presence of a direct dye

\begin{tabular}{|c|c|c|c|c|c|c|}
\hline \multirow{2}{*}{$\frac{\text { Sample }}{\mathrm{MC}}$} & \multicolumn{6}{|c|}{$2 \theta / \mathrm{deg}$} \\
\hline & & & $14.5(w)$ & $16.8(w)$ & $20.4(w)$ & $22.7(\mathrm{~s})$ \\
\hline DB71 & & & & $16.8(w)$ & & $23.5(\mathrm{~s})$ \\
\hline DBr106 & $9.0(\mathrm{vw})$ & $10.8(\mathrm{vw})$ & & & & $23.0(\mathrm{~s})$ \\
\hline DR28 & & $11.0(\mathrm{w})$ & & & & $23.0(\mathrm{~s})$ \\
\hline DR80 & $9.0(\mathrm{vw})$ & $12.0(\mathrm{vw})$ & & & $20.6(\mathrm{~s})$ & \\
\hline
\end{tabular}

to those of products obtained in the presence of various direct dyes. ${ }^{8,12,14-16}$ The diffractogram of the product suggests that all direct dye diffuse into nascent $\mathrm{MC}$ and form the characteristic complex composed of a dye and cellulose instead of the ordinary structure of MC.

The diffractograms of uniplanar-orientated membranes of DBr106, DB71, DR28, and 80 products measured by transmission and reflection methods are shown in the Figure 3. $2 \theta$ s of the diffraction angle of each product are listed in Table II.

When the DB71 product membrane is measured by reflection, a strong and a weak diffraction peaks occur at $8.6^{\circ}$ and $16.6^{\circ}$, respectively, whereas, in case of transmission, strong and weak peaks occur at $23.5^{\circ}$ and $16.7^{\circ}$, respectively. This shows that the diffraction plane near $16.7^{\circ}$ does not attain uniplanar-orientation. In the reflection diffractogram of the $\mathrm{DBr} 106$ product membrane, strong and very weak peaks occur at $10.8^{\circ}$ and $22.5^{\circ}$, whereas, in transmission, strong and weak peaks occur at $23.0^{\circ}$ and $10.5^{\circ}$, respectively. Similar uniplanarorientation was observed in the case of the DR28 product

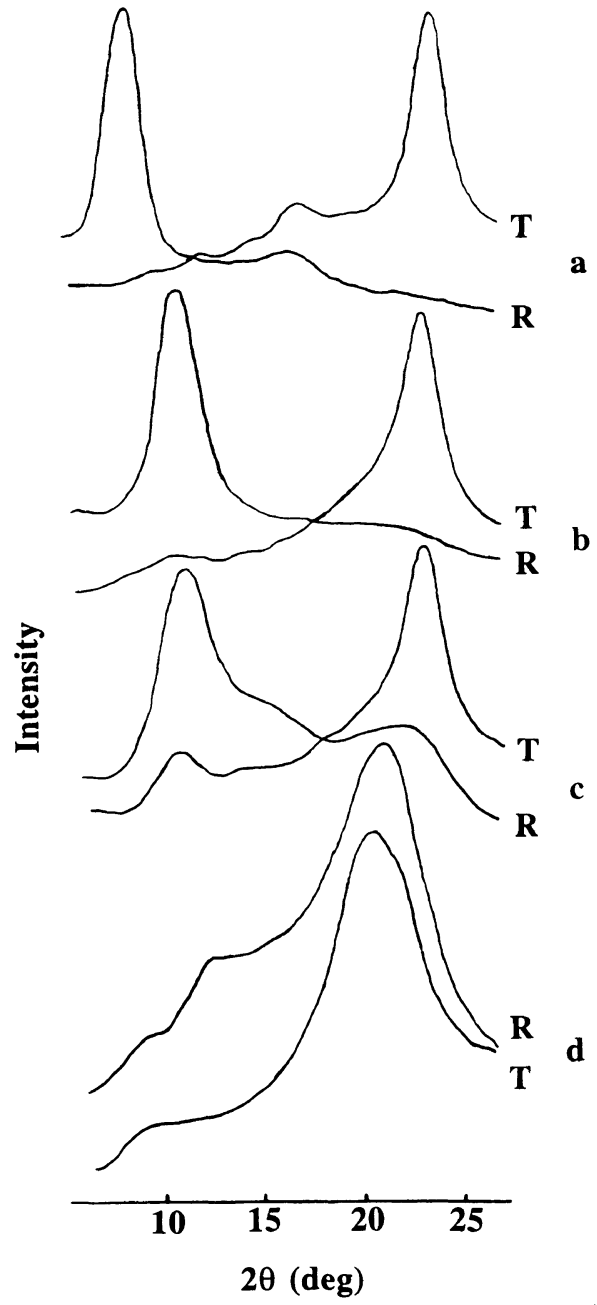

Figure 3. X-Ray diffractogams of oriented membrane of product samples measured by transmission (T) and reflection (R). a, DB71 product; b, DBr106 product; c, DR28 product; d, DR80 product.

Table II. $2 \theta$ values of diffraction peaks in diffractograms of oriented membrane of the products measured by transmission and reflection methods

\begin{tabular}{|c|c|c|c|c|c|c|}
\hline \multirow{2}{*}{$\frac{\text { Dye }}{\text { DB71 }}$} & \multirow[b]{2}{*}{$\mathrm{R}$} & \multicolumn{5}{|c|}{$2 \theta / \mathrm{deg}$} \\
\hline & & $8.6(\mathrm{~s})$ & & $16.6(w)$ & & \\
\hline & $\mathrm{T}$ & - & & $16.7(w)$ & & $23.5(\mathrm{~s})$ \\
\hline \multirow{2}{*}{ DBr106 } & $\mathrm{R}$ & & $10.8(\mathrm{~s})$ & & & - \\
\hline & $\mathrm{T}$ & & $10.5(\mathrm{vw})$ & & & $23.0(\mathrm{~s})$ \\
\hline \multirow[t]{2}{*}{ DR28 } & $\mathrm{R}$ & & $11.2(\mathrm{~s})$ & & & $23.0(\mathrm{w})$ \\
\hline & $T$ & & $10.7(\mathrm{w})$ & & & $23.0(\mathrm{~s})$ \\
\hline \multirow[t]{2}{*}{ DR80 } & $\mathrm{R}$ & $9.0(\mathrm{vw})$ & $12.4(w)$ & & $21.9(\mathrm{~s})$ & \\
\hline & $\mathrm{T}$ & $9.0(\mathrm{vw})$ & - & & $20.0(\mathrm{~s})$ & \\
\hline
\end{tabular}

membrane. The diffratograms of three products resemble that of the product obtained in the presence of Fluorescent Brightener as reported previously. ${ }^{8}$ In contrast, in both the reflection and transmission diffractograms of the DR80 product, strong and weak peaks occur near $21^{\circ}$ and $9.0^{\circ}$, respectively. The diffractogram of the DR80 product resembles those of DR75 and 79 products. ${ }^{16}$

The diffraction plane at the low angle side of DB71, DBr106, or DR28 product membrane parallels the surface of the membrane, whereas the diffraction plane at the high angle side is perpendicular to the surface of the membrane. The behavior of the uniplanar orientation of these product membranes is similar to that of the 


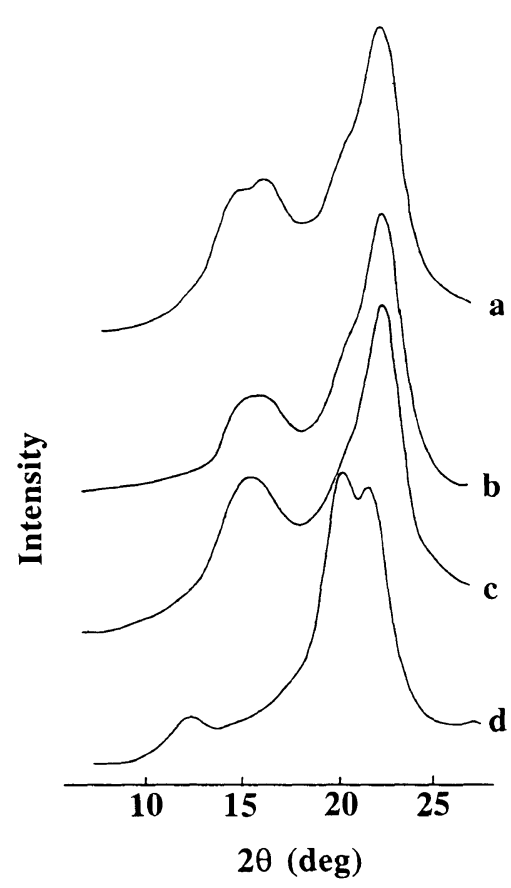

Figure 4. X-Ray diffractograms of cellulose regenerated from product samples. a, DB71 cellulose; b, DBr 106 cellulose; c, DR28 cellulose; d, DR80 cellulose.

Table III. X-Ray diffraction angles of cellulose I, II, IV and cellulose regenerated from product samples

\begin{tabular}{lccc}
\hline \multirow{2}{*}{ Sample } & \multicolumn{3}{c}{$2 \theta / \mathrm{deg}$} \\
\cline { 2 - 4 } & $(1 \overline{1} 0)$ & $(110)$ & $(020)$ \\
\hline MC & 14.5 & 16.8 & 22.7 \\
DB71 & 14.5 & 16.3 & 22.6 \\
DBr106 & 15.1 & 16.3 & 22.4 \\
Cellulose IV & 15.4 & 15.4 & 22.4 \\
DR28 & 15.4 & 15.4 & 22.4 \\
Cellulose II & 12.0 & 19.8 & 21.1 \\
DR80 & 12.2 & 20.4 & 21.7 \\
\hline
\end{tabular}

product membrane obtained in the presence of Fluorescent Brightener. ${ }^{8}$ The strong diffraction peak at the low angle side suggests that spacing is due to the inclusion of a dye between the cellulose sheets corresponding to the (110) plane of MC. The DR80 product membrane does not show such uniplaner-orientation. This is due to the removal of dye from the complex during rinsing of the product sample.

Figure 4 shows the $\mathrm{X}$-ray diffractograms of celluloses (DB71 cellulose, DBr106 cellulose, DR28 cellulose, and DR80 cellulose) regenerated from the product by dyeextraction. $2 \theta \mathrm{s}$ of each diffraction peak are listed in Table III.

As apparent from Figure 4 and Table III, the diffractograms of DB71 and DBr106 celluloses are almost the same as that of cellulose I. The diagram of the DR28 cellulose coincides with that of cellulose IV. The diagram of DR80 cellulose is similar to that of cellulose II. According to the characteristics of each direct dye, the regenerated cellulose forms specific crystals.

The fine structure of regenerated cellulose seems to be produced in the process of sorption and desorption of a direct dye. The degree of interaction between a direct
Table IV. Molecular weights of dyes

\begin{tabular}{lrccclc}
\hline Dye & MW & NH & NS & MW/N & Crystal type & Ref \\
\hline FB & 916 & 8 & 2 & 458 & Cellulose I & 7 \\
DBr106 & 1198 & 2 & 4 & 300 & Cellulose I & a \\
DB71 & 941 & 2 & 4 & 235 & Cellulose I & a \\
DR28 & 812 & 2 & 2 & 406 & Cellulose IV & $13^{\text {a }}$ \\
DR79 & 948 & 4 & 4 & 237 & Cellulose II & 17 \\
DR75 & 902 & 6 & 4 & 226 & Cellulose II & 17 \\
DB1 & 904 & 4 & 4 & 226 & Cellulose II & 15 \\
DB14 & 872 & 4 & 4 & 218 & Cellulose II & 16 \\
DR80 & 1236 & 4 & 6 & 206 & Cellulose II & a
\end{tabular}

NS: Number of sulfonate, NH: Number of group with hydrogen bonding ability, MW: Molecular weight, N: Number. ${ }^{a}$ Present work.

dye and cellulose chain depends on the planarity of a dye molecule, straightness, length of the conjugated double bond, proton donor or acceptor. ${ }^{18}$ Although there are no differences in the planarity and straightness of four dyes, the length of conjugated double bond, groups having the amount of the hydrogen bonding ability, the number of sulfonate group differs. The order of length of the conjugated double bond is $\mathrm{DBr} 106>$ DR $80>$ DB71 $>$ DR28. In case of the number of the group having the hydrogen bonding ability, DR80 has four, DR28, DB71, and DBr 106 have two except for the sulfonate group, respectively. In case of the sulfonate group, DR80 has six, DBr106 and DBu71 have four, and DR28 has two.

As cellulose I is regenerated from DBr106 and DB71 products, it seems that these dyes do not have influence on conformation of cellulose chains. However, cellulose regenerated from the complex including a dye between the cellulose sheets does not form cellulose $\mathrm{I} \alpha$ rich but forms cellulose I $\beta$ rich crystal. ${ }^{11}$ This suggests that these dyes influence only the arrengement of cellulose chains. Similarly, that cellulose $\mathrm{IV}_{\mathrm{I}}$ is regenerated from the DR28 product ${ }^{13}$ indicates the conformation of cellulose chains does not change, ${ }^{19}$ but the arrengement changes. DR80 influences conformation and arrengement of cellulose chains during regeneration and hence, regenerated cellulose is cellulose II.

The length of the conjugated double bond and number of groups having hydrogen bonding ability may not be the factor determining the crystal type of regenerated cellulose. Although the number of sulfonate group in a dye is not concerned apparently, it is assumed that the molecular weight per sulfonate group has great influence on crystal type (Table IV). Cellulose II is regenerated from the product obtained in the presence of a direct dye with molecular weight less than 300 per sulfonate group and in case of more than 300 , cellulose $I$ or $\mathrm{IV}_{\mathrm{I}}$ is regenerated. In the case of DB71, the result is exceptional.

Although the affinity of the direct dye toward cellulose might be determined by various factors, the influence of the sulfonate group on the conformation of cellulose chains seems predominant.

\section{REFERENCES}

1. F. Horii, A. Hirai, and R. Kitamaru, Macromolecules, 20, 2117 (1987).

2. A. Kai and T. Koseki, Makromol. Chem., 186, 2609 (1985). 
3. J. R. Colvin, M. Takai, L. C. Sowden, and J. Hayashi, Int. J. Biol. Macromol., 4, 244 (1982).

4. A. Kai and J. Kogusuri, Nippon Kagaku Kaishi, 198 (1982).

5. A. Kai, Makromol. Chem., Rapid Commun., 5, 307 (1984).

6. A. Kai, Makromol. Chem., Rapid Commun., 5, 653 (1984).

7. A. Kai and P. Xu, Polym. J., 22, 955 (1990).

8. A. Kai and P. Xu, Kobunshi Ronbunshu, 48, 449 (1990).

9. C. H. Haigler, R. M. Brown, and M. Benziman, Science, 210, 903 (1980).

10. M. Benziman, C. H. Haigler, A. R. White, and K. M. Copper, Proc. Natl. Acad. U.S.A., 77, 6678 (1980).

11. A. Kai, P. Xu, F. Horii, and S. Hu, Polymer, 35, 75 (1994).
12. A. Kai, Y. Kido, and N. Ishida, Chem. Lett., 949 (1990).

13. A. Kai and Md. I. H. Mondal, Int. J. Biol. Macromol., 20, 221 (1997).

14. Md. I. H. Mondal and A. Kai, Polym. J., 30, 78 (1998).

15. Md. I. H. Mondal and A. Kai, Polym. J., 30, 84 (1998).

16. A. Kai and Md. I. H. Mondal, Polym. J., 30, 398 (1998).

17. S. Hestrin and M. Schramm, Biochem. J., 58, 345 (1954).

18. N. Kuroki, in "Theoretical Chemistry of Dyeing," Maki-shoten, Tokyo, 1966, p 149.

19. J. Hayashi, H. Kon, M. Takai, M. Hatano, and T. Nozawa, in "The Structures of Cellulose," R. H. Atalla, Ed., ACS Symposium Series 340, Washington, D.C., 1985, p 135. 